\title{
Image Enhancement Algorithm Based on Improved Lateral
}

\section{Inhibition Network}

\author{
Haijiao Yun ${ }^{\mathrm{a}, \mathrm{b}^{*}}$, Zhiyong Wu ${ }^{\mathrm{a}}$, Guanjun Wang ${ }^{\mathrm{a}, \mathrm{b}}$, Gang Tong ${ }^{\mathrm{a}}$, and Hua Yang ${ }^{\mathrm{a}}$ \\ ${ }^{a}$ Changchun Institute of Optics, Fine Mechanics and Physics, Chinese Academy of Sciences, Changchun, Jilin, \\ 130033, China \\ ${ }^{\mathrm{b}}$ University of Chinese Academy of Sciences, Beijing, 100049, China
}

\begin{abstract}
There is often substantial noise and blurred details in the images captured by cameras. To solve this problem, we propose a novel image enhancement algorithm combined with an improved lateral inhibition network. First, we built a mathematical model of a lateral inhibition network in conjunction with biological visual perception; this model helped to realize enhanced contrast and improved edge definition in images. Next, we proposed that the adaptive lateral inhibition coefficient adhere to an exponential distribution thus making the model more flexible and more universal. Finally, we added median filtering and a compensation measure factor to build the framework with high pass filtering functionality thus eliminating image noise and improving edge contrast, addressing problems with blurred image edges. Our experimental results show that our algorithm was able to eliminate noise and the blurring phenomena and enhance the details of visible and infrared images.
\end{abstract}

Keywords: image enhancement, lateral inhibition network, adaptive lateral inhibition coefficient, compensation measure factor.

* Corresponding Author, E-mail: yunhaijiao2011@126.com

\section{Introduction}

Image enhancement is an indispensable technique and essential method of improving image quality in digital image processing. Even with the ubiquity of digital cameras and mobile telephones, there are substantial amounts of noise in images because of camera defocusing, a lack of uniform illumination, atmospheric disturbances, and the like not clear edge texture and producing dark or highlight area, i.e., which have a great impact on executing missions. Hence, it 
is necessary and useful to develop an effective enhancement algorithm that addresses such noise in digital images.

To remove noise from images, many denoising methods based on the features of the images, the characteristics of the noise, and spectrum distribution are proposed by scholars. They can be broadly divided into three categories: denoising methods in area space, such as mean filtering, median filtering, i.e.; denoising methods in frequency space, such as homomorphic filtering, i.e.; as well as the donising methods based on sparse representation, such as the block-match and 3D filtering, BM3D, i.e.. ${ }^{[1-6]}$ Using the classical methods of image denoising, the noise was effectively removed, but the image edge easily appeared fuzzy phenomenon. In recent years, scholars worldwide have developed a variety of image enhancement algorithms from the perspective of image characteristics and various mathematical theories; ${ }^{7-8}$ however, the actual visual effect is not necessarily favorable without also considering the visual features. To handle image information, scholars have referenced the principles of visual lateral inhibition as early as $1980 .^{9}$

In this early research, background information of images was extracted from high levels of background interference. In subsequent research, scholars have achieved image edge detection, edge-sharpening, and enhanced contrast via the theory of lateral inhibition. ${ }^{10-12}$ With the further development of research, scholars proposed numerous enhancement algorithms based on various 
parameters of the lateral inhibition network and the characteristics of image quality. ${ }^{13-17}$ Some scholars proposed enhancement algorithms that combined a lateral inhibition network with adaptive filtering. Using such an approach, the system adaptively selects model parameters according to different local variances and averages of images, thus eliminating noise.

Based on the above, we proposed a new improved algorithm that incorporates a lateral inhibition network, which is equivalent to a high pass filter and sensitive to noise. Furthermore, we introduce a compensation measure factor that causes the network to enhance the edges and remove noise, thus solving the problem of image edge fuzziness. Our experimental results show that our proposed method effectively removes the noise from images and improves the quality of images.

The rest of this letter is organized as follows. Section 2 overviews the lateral inhibition network theory. Then we present the details of the proposed method to enhance images in the Sec. 3. The experimental results are given in Sec. 4. Finally, a conclusion is drawn in Sec. 5.

\section{Lateral Inhibition Network Theory}

Lateral inhibition was discovered and confirmed by the Limulus visual physiological electrical experiments. ${ }^{[16]}$ Here, every Limulus eye is considered as a single receptor. When the center receptor receives a slice of strong light stimulus, the excitability of its surrounding receptors will 
be inhibited, and vice versa. That is called the lateral inhibition phenomenon in that the Limulus eyes produce conditionality in each other. Suppose the two receptors A, B do the experiments, assuming that the A and B are respectively lighted, whose light emitting powers are $g_{\mathrm{A}}$ and $g_{B}$, respectively; when the A and B are simultaneously lighted, detecting their light emitting powers are reduced to $f_{\mathrm{A}}$ and $f_{\mathrm{B}}$, respectively; meanwhile, the stronger endured the degree of light stimulus by $\mathrm{A}$, the smaller emitted the pulse frequency by B. That shows the receptor A is inhibited by the receptor B; on the other hand, the receptor B is also inhibited by the receptor A, called the lateral inhibition. The lateral inhibition effect can be described as:

$$
\begin{aligned}
& g_{\mathrm{A}}=f_{\mathrm{B}}-k_{\mathrm{BA}}\left(f_{\mathrm{B}}-f_{\mathrm{AB}}\right) \\
& g_{\mathrm{B}}=f_{\mathrm{B}}-k_{\mathrm{AB}}\left(f_{\mathrm{A}}-f_{\mathrm{BA}}\right)
\end{aligned}
$$

where, $g_{\mathrm{A}}$ and $g_{B}$ represent light emitting powers of the two receptors by single light, respectively; $f_{\mathrm{A}}$ and $f_{B}$ represent light emitting powers of the two receptors with the lateral inhibition effect, respectively; $k_{\mathrm{AB}}$ and $k_{\mathrm{BA}}$ represent the lateral inhibition coefficients between two receptors; $f_{\mathrm{AB}}$ and $f_{\mathrm{BA}}$ represent the threshold value of the lateral inhibition.

Hence, we can find that the neighboring receptors' light emitting power below the threshold value doesn't produce lateral inhibition effect; besides the lateral inhibition coefficient's value depends on the distance between two receptors. In the lateral inhibition network composed of $\mathrm{n}$ receptors, the pth receptor is endured inhibition effect from surrounding receptors, at this moment the Eq. (1) is extended: 


$$
g_{p}=f_{p}-\sum_{j=1}^{n} k_{j p}\left(f_{j}-f_{p j}\right) \quad p=1,2, \ldots, n ; j=1,2, \ldots, n ; p \neq j(2)
$$

According to this principle, the receptors in the bright and dark border are inhibited, then

producing noticeable bright and dark lines over there. Hence, this lateral inhibition phenomenon helps to enhance information extraction of the retina, increasing both contour and contrast, thus making targets much clearer and easier to recognize.

\section{Image Enhancement Model Based on Lateral Inhibition Networks}

\subsection{Enhancement Model}

In order to make one-dimensional lateral inhibition network applied to image processing, it must be extended to that of two-dimensions. The traditional lateral inhibition algorithm adopted a nonrecurrent lateral inhibition network model with ignoring the threshold value, ${ }^{18}$ which can be expressed as

$$
G(m, n)=F(m, n)-\sum_{i=-l}^{l} \sum_{j=-l}^{l} k(i, j) \cdot F(m+i, n+j),
$$

where $F(m, n)$ represents the input image, $G(m, n)$ represents the output image, $k(i, j)$ represents the inhibition coefficients matrix, and 1 is the inhibition field.

The traditional lateral inhibition network is sensitive to noise. The method used in [18] adopts a lateral inhibition network combined with a mean filtering to remove noise, which disperses the grayscale values of noise among the surroundings to realize smoothness; however, 
images often become fuzzy as a result. Compared with mean filtering, median filtering results in a better smoothness and clearer contour. Therefore, we process images with a lateral inhibition network combined with median filtering to remove noise, which is expressed as:

$$
\begin{aligned}
& G(m, n)=\overline{F(m, n)}-\sum_{i=-l}^{l} \sum_{j=-l}^{l} k(i, j) \cdot F(m+i, n+j), \\
& \overline{F(m, n)}=\underset{(r, s) \in(-l, i, i)}{\operatorname{median}}\left[F\left(k_{1}+r, k_{2}+s\right)\right] .
\end{aligned}
$$

where, $F(m, n)$ represents $F(m, n)$ 's median value in its neighborhood and l' is the size of the median filtering window.

To remove noise and avoid edge fuzziness, we introduce a compensation measure factor to suppress noise signal. When it is greater than a given threshold value, considered as a valid signal mutation and carried on enhancing this signal; and vice, considered as a noise mutation and carried on inhibiting noise. For one-dimensional signal with noise, if there is signal intensity mutation near the mutational site, the signal intensity has some correlation each other; if there is signal intensity mutation near the noise point, the signal intensity has no correlation each other. The signal average intensity is not equal located in the mutational site on both sides, but the signal average intensity is very approximate located in the noise point on both sides. For the images, the edge points are grayscale mutational sites with a certain direction, and define a direction perpendicular to the grayscale mutational site as edge direction. Hence, most can be found at least one edge point along the edge directions in any neighborhood of the edge points. 
Using the edge points along the edge direction, the neighborhood pixels can be divided into at least two sub-regions, whose internal grayscales are homogeneous distribution. However, the difference of grayscale is larger between the two sub-regions; but there is not this feature for the noise in the smooth region.

The compensation measure factor processes pixels by distributing into the four pairs of subregions shown in Fig. 1. The neighborhood of every pixel in the image is divided into two equal parts, each of which is processed using median filtering and a $5 \times 5$ template. On each subsidiary neighborhood, the number of pixel $(m, n)$ is respectively $t_{1}$ and $t_{2}$, with grayscale values of pixels is $\mathrm{p}_{1 \mathrm{i}}$ and $\mathrm{p}_{2 \mathrm{i}}$, respectively. Hence, the respective grayscale averages of each subsidiary are

$$
\begin{aligned}
& \mu_{1}=\frac{1}{t_{1}} \sum_{i=1}^{t_{1}} p_{1 i}, \\
& \mu_{2}=\frac{1}{t_{2}} \sum_{i=1}^{t_{2}} p_{2 i} .
\end{aligned}
$$

Our method consists of three steps, each step is summarized below.

Step 1 . We compute the grayscale mutation values of pixel $(\mathrm{m}, \mathrm{n})$ 's subsidiary in the four directions, i.e.,

$$
\Delta g_{s}=\left|\mu_{1}-\mu_{2}\right|_{s}, \quad s=1,2,3,4 .
$$

Step 2. Set $\Delta g(m, n)=\max _{s=1}^{4} \Delta g_{s}$, and set $\Delta g_{\max }=\max _{m, n} \Delta g(m, n)$.

Step 3. Pixel $(\mathrm{m}, \mathrm{n})$ is potentially an edge point if it meets the following requirements:

$$
E(m, n)=\Delta g(m, n) / \Delta g_{\max }, \quad 0 \leq E(m, n) \leq 1 . \text { (9) }
$$

The larger $\mathrm{E}(\mathrm{m}, \mathrm{n})$ is the more likely it is an edge point, but also, the more likely it is noise. 


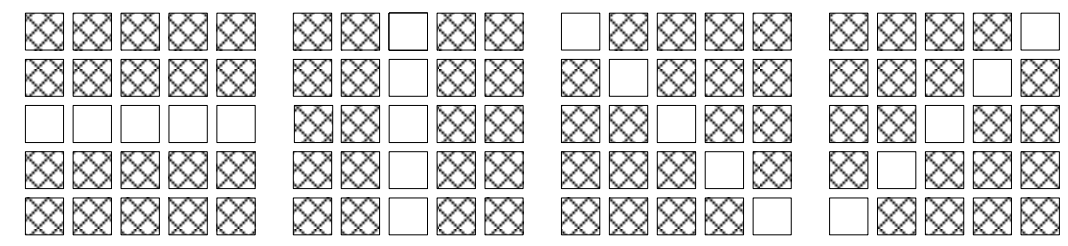

Fig. 1 Four defined sub-regions of pixels.

Introducing the compensation measure factor, the lateral inhibition network model is improved as

$$
G(m, n)=\overline{F(m, n)}-\sum_{i=-l}^{l} \sum_{j=-l}^{l} k(i, j) \cdot F(m+i, n+j)+E(m, n) \times(F(m, n)-\overline{F(m, n)}) .
$$

This improved network can distinguish from the noise and edge points to a certain extent, i.e., causing high-brightness points of the edge to be relatively brighter and low-brightness points are relatively darker. Furthermore, the improved network improves the contrast of the edge points, suppressing noise at the same time.

\subsection{Lateral inhibition coefficient distribution}

According to biological visual perception, the lateral inhibition coefficient is inversely proportional to the distance between the two receptors. An anisotropic filtering method proposed by SHI can determine inhibition coefficients according to scene, ${ }^{19}$ but the lateral inhibition

coefficient distribution must satisfy a Gaussian function whose expression is overly complex..$^{20,21}$ Hence, we propose an exponential lateral inhibition coefficient distribution. Here, the exponential function is a monotone function and has rapid attenuation with the increase of an independent variable, which is similar to the relationship of the lateral inhibition network and 
distance between the two different receptors. The alterative tendency of the lateral inhibition coefficient distribution is shown in Fig. 2, and is expressed as

$$
\begin{aligned}
& k=A \exp \left(-\frac{d_{i j, p q}}{\rho}\right), \\
& \rho=\frac{1}{F(m, n)} .
\end{aligned}
$$

here, $\mathrm{A}$ is a constant. Eq. (11) is based on the definition of the Euclidean distance formula, $d_{i j, p q}=\sqrt{(i-p)^{2}+(j-q)^{2}}$, which represents distance between the central receptor $(\mathrm{i}, \mathrm{j})$ and the other surrounding receptor $(\mathrm{p}, \mathrm{q})$ in the same inhibition field. In Eq. (12), F (m, n) represents pixel (m, n)'s grayscale value.

Assuming that A has been set in Eq. (11), the different values of $\rho$ determine the decay rate of the exponential function, as shown in Fig. 2.

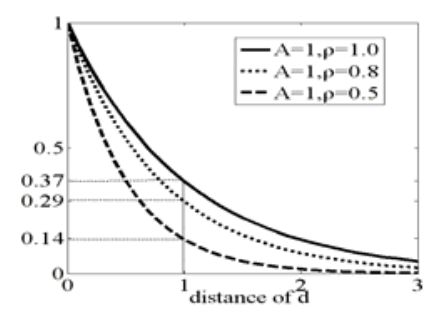

Fig. 2 Exponential function's decay rate based on different values of $\rho$

From Fig. 2, we observe that the inhibition coefficient of $\mathrm{k}$ is larger when $\rho$ is larger at the same distance of $d$. For example, the background grayscale value is often smaller than the target grayscale value of the infrared image; in such cases, the infrared background is inhibited more intensely and infrared target is inhibited less intensely. Thus, the image target can be more 
clearly distinguished from the image background. Overall, this satisfies the characteristics of the lateral inhibition network, which can enhance the contrast and stand out the edges.

\subsection{Lateral inhibition field selection}

The lateral inhibition field refers to the maximum distance between the central receptor and its surrounding other receptors. Based on on-type of biological vision, ${ }^{17,22}$ nerve cells emit a charge under light stimulation. The amount of the emitting electronic signal is proportional to light stimulation in the lateral inhibition field, which benefits the edge extraction effect. According to necessary degree of inhibition intensity, the inhibition fields may be $3 \times 3,5 \times 5,7 \times 7,11 \times 11$ or larger. We used our proposed method to process the infrared image at the different lateral inhibition fields, as shown in Fig. 3 below.

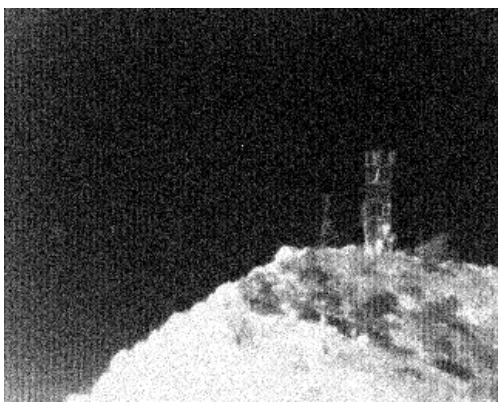

(a)

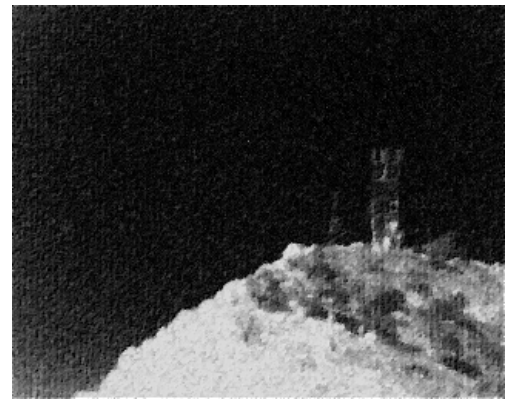

(b)

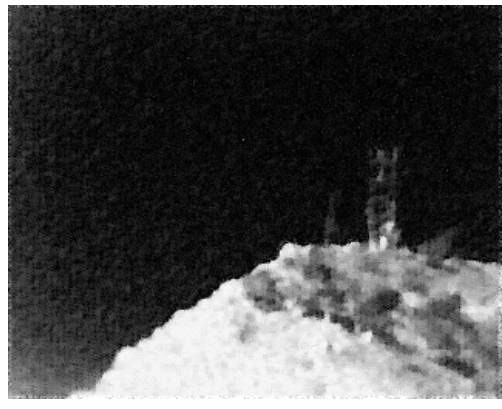

(c) 


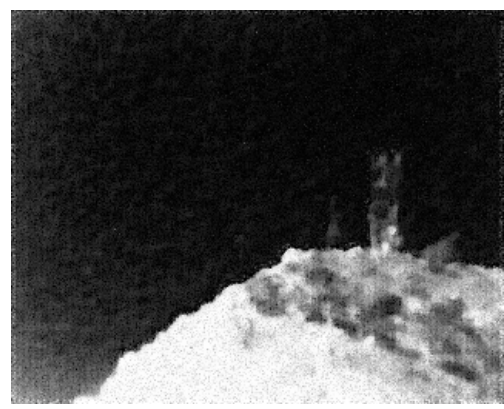

(d)

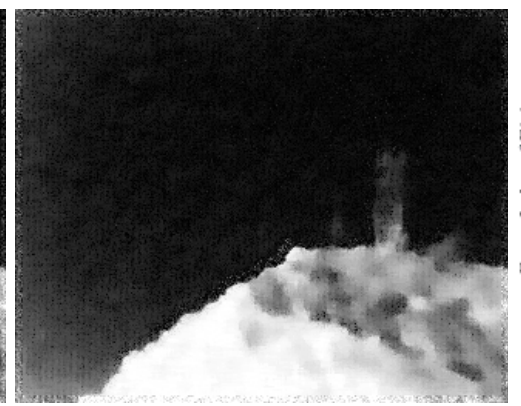

(e)

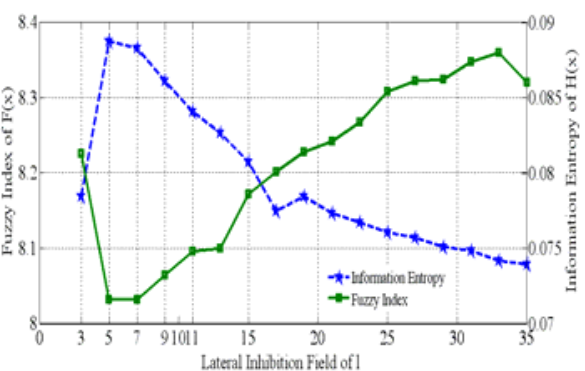

(f)

Fig.3 Comparison of enhanced infrared images at the different lateral inhibition fields. (a) original noisy image; (b)

with a lateral inhibition field of $3 \times 3$; (c) with a lateral inhibition field of $5 \times 5$; (d) with a lateral inhibition field of 7

$\times 7$; (e) with a lateral inhibition field of $11 \times 11$; and (f) bi-axes diagram of the information entropy and fuzzy index

at the different lateral inhibition fields.

From Figs. 3(b)-3(e), we observe that infrared image noise was gradually reduced with the increase of the lateral inhibition field, but the fuzzy phenomenon become more pronounced. Fig. 3(f) shows the bi-axes diagrams of the information entropy and fuzzy index values of the enhanced images at the different lateral inhibition fields. We observe here that we can obtain the best fuzzy index and information entropy values with an inhibition field of $5 \times 5$.

Our experimental results show that enhanced images are not obviously enhanced when small inhibition fields are used; however, enhanced images are severely anamorphic and computationally expensive when large inhibition fields are used. Hence, we note that it is best to 
enhance images with an inhibition field of $5 \times 5$, which achieves a practical compromise of computation and noticeable enhancements.

\section{Experimental results and analysis}

\subsection{Comparing image visual effects}

In our experiments, the programming platform is Matlab R2012a, and the original images are added Gaussian noise, whose variance is 0.03 , obtaining the noise images. There are three kinds of test images, including infrared images of size 320 pixel $\times 256$ pixel, visible light images of size 512 pixel $\times 320$ pixel, and natural images of size 768 pixel $\times 512$ pixel. The first two images are captured from the photoelectric theodolite in the shooting range. The third images are obtained from LIVE Image Quality Assessment Database Release 2. ${ }^{23}$ All three kinds of images were converted into grayscale images. Furthermore, we used two-dimensional histogram equalization $(2 \mathrm{DHE}),{ }^{24}$ two-dimensional median filtering $(2 \mathrm{DMF}),{ }^{25}$ the block-match and $3 \mathrm{D}$ filtering (BM3D), the method proposed in [18], and our proposed method to process all test images. The original and enhanced images for each of the three test images are shown in Figs. $4-6$. To obtain a higher image quality and better visual effect, we determined via a large number of experimental results that parameter $\mathrm{A}$ was best set at $0.6 .^{[18]}$ As noted above, we selected an inhibition field of $5 \times 5$ because of the required amount of computation. 
Test 1. Fig. 4 shows the resulting images for the infrared image obtained by both the conventional methods and our proposed method. The input image with noise is shown in Fig. 4(a). The converted images obtained by 2DHE, 2DMF, BM3D, the method proposed in [18], and our proposed method are shown in Figs. 4(b)-4(f), respectively. We observe that 2DHE effectively improved image contrast, but noise still exits, with the visibility of characters on the boat worsening. We conclude that the image quality of Fig. 4(b) is poor.

Next, we note that 2DMF successfully removed noise from the entire image, as shown in Fig. 4(c). The resulting image contained fuzziness and had low contrast, all indicating that an excessive enhancement of the boat occurred. The BM3D method makes the noise images sparse representation with the dictionary obtained from training the similar noise images. This enhancement method achieved better denoising effect, but the method is easy to weaken the texture and produce edge fuzziness and the block effect in Fig. 4(d). Such over-enhancement was also observed in Fig. 4(e), which is the resulting image obtained via the method proposed in [18]. In the image, the hull is completely blurred. Furthermore, the resulting image was not enhanced from the perspective of image contrast. Overall, the image quality is insufficient. Finally, the enhanced image obtained by our proposed method shown in Fig. 4(f) is visually appealing. Image noise and contrast were improved with a good balance. 


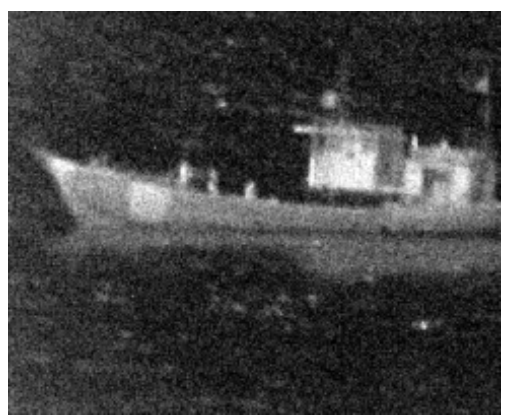

(a)

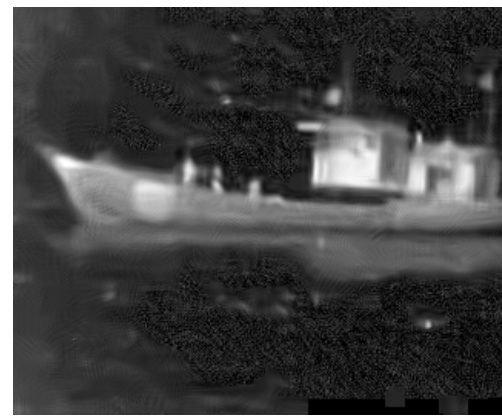

(d)

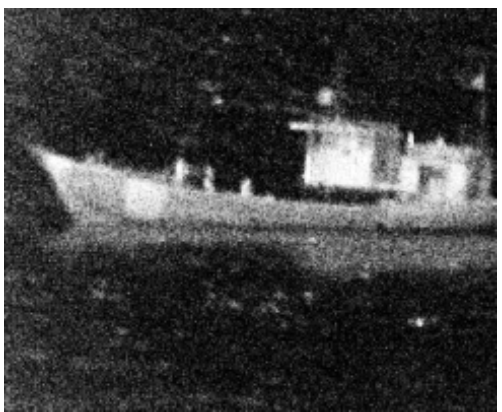

(b)

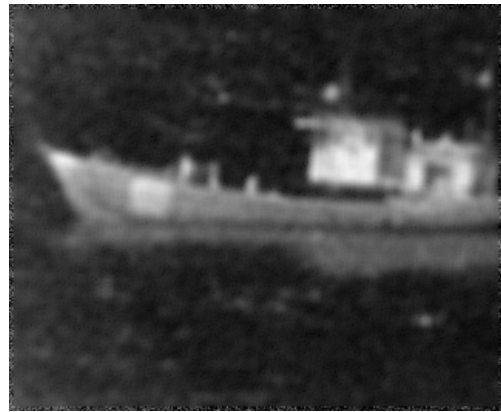

(e)

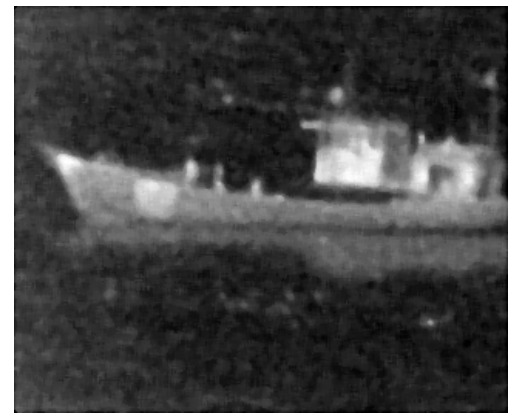

(c)

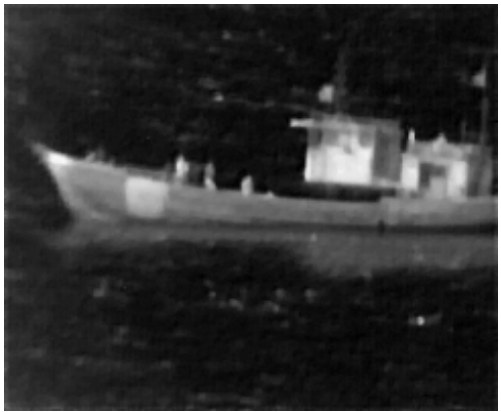

(f)

Fig.4 Comparisons of enhanced images obtained from the infrared camera: (a) original input image; (b) 2DHE; (c)

2DMF; (d) BM3D; (e) the method proposed in [18]; and (f) our proposed method

Test 2. Fig. 5 shows the resulting images based on a visible light image obtained from optoelectronic theodolite in the same manner as Fig. 4. In the 2DHE result, shown in Fig. 5(b), there is still noise and the intensity of image enhancement is not large. The images showing the effect of 2DMF and the method proposed in [18] are good, as shown in Fig. 5(c) and 5(e), respectively; however, the contrast enhancement effect of both $2 \mathrm{DMF}$ and the method proposed in [18] is insufficient. Furthermore, the fuzziness phenomenon is serious. Meanwhile, the block effect is very noticeable in the enhanced images by the BM3D method in Fig. 5(d). From Fig. 
5(f), we observe that our proposed method was able to make up for these shortages. The contrast and texture details of the enhanced image are obviously improved and more useful for recognize the image's content.

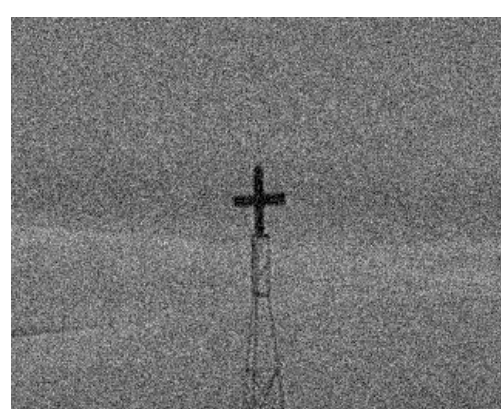

(a)

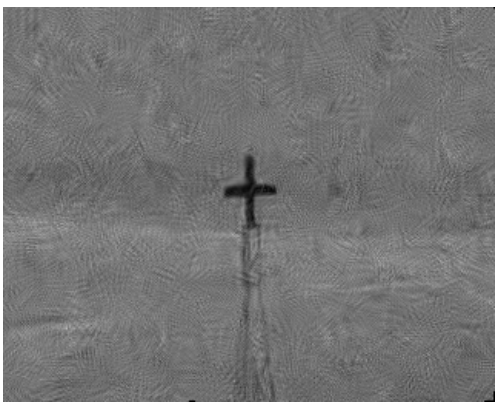

(d)

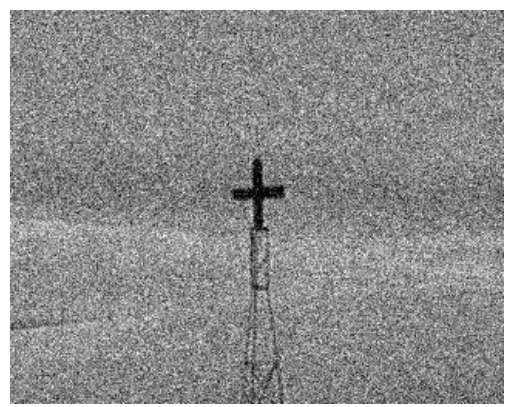

(b)

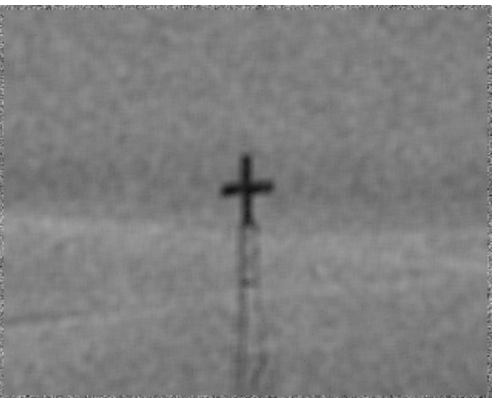

(e)

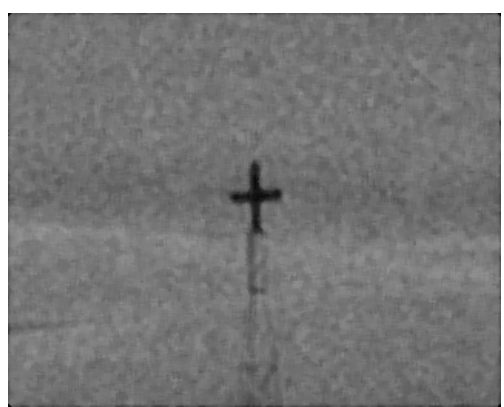

(c)

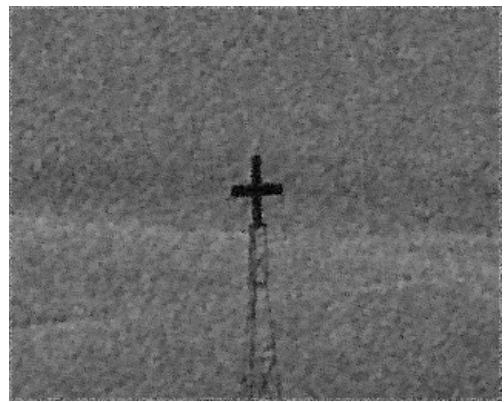

(f)

Fig.5 Comparison of enhanced images obtained from the visible light camera: (a) original input image; (b) 2DHE;

(c) 2DMF; (d) BM3D; (e) the method proposed in [18]; and (f) our proposed method

Test 3. Fig. 6 shows the resulting images of natural image obtained from LIVE laboratory. Here, as shown in Fig. 6(b), 2DHE obtained superior results as compared with the enhancements for the infrared and visible light images; however, the removal of noise in 2DHE is poor in comparison with the other four methods. The impression received from the resulting image 
obtained by 2DMF, as shown in Fig. 6(c) is that it is insufficient, though it is better than that of the case of removing noise. The denoising effect of the BM3D method is better than first two conventional methods, but this method still exists the block effect to debase enhanced image quality, as shown in Fig. 6(d). The contrast enhancement effect of the method proposed in [18] is also insufficient, resulting in an image that gives a fuzzy impression, as shown in Fig. 6(e), though the removal of noise is performed well. The image obtained by our proposed method, shown in Fig. 6(f), is better than the image obtained by the method proposed in [18] in terms of image contrast and noise, and giving an impression of liveliness. From these results, we conclude that our proposed method is superior to the others.

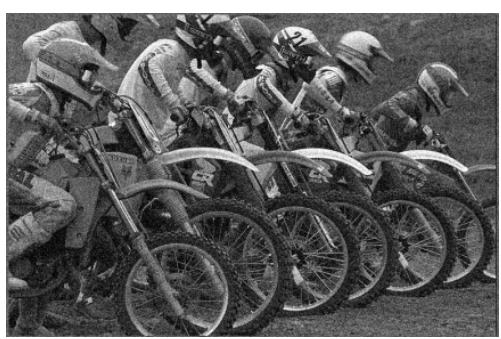

(a)

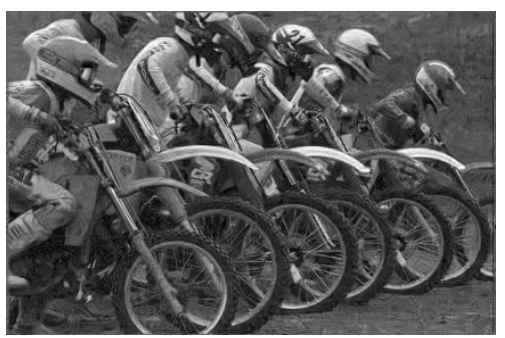

(d)

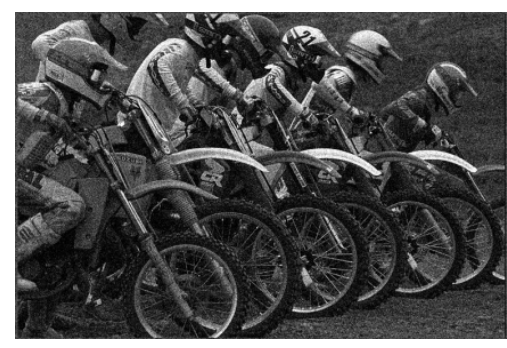

(b)

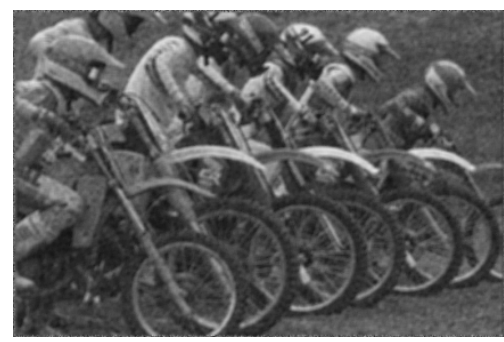

(e)

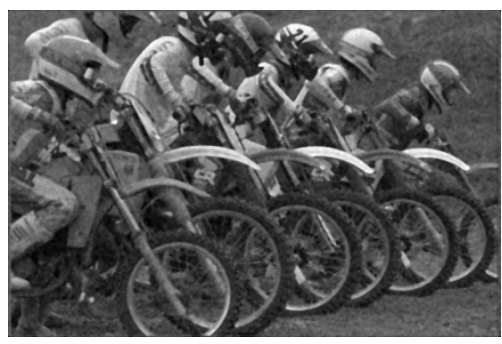

(c)

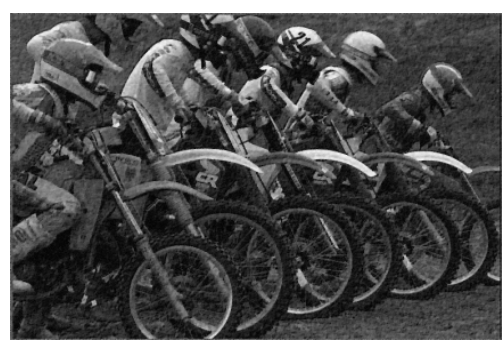

(f) 
Fig.6 Comparison of enhanced images obtained from LIVE laboratory: (a) original input image; (b) 2DHE; (c)

2DMF; (d) BM3D; (e) the method proposed in [18]; and (f) our proposed method

Test 4. Test the algorithms' processing time, comparing with the performance and efficiency among the five algorithms. The CPU for test of the personal computer is Intel Core(TM)2 Duo Q9550, whose frequency is $2.83 \mathrm{GHz}$, memory is $3.5 \mathrm{~GB}$, and programming platform is DELL Windows XP + Matlab R2012a. There are three kinds of test images, which are the infrared images of size 320 pixel $\times 256$ pixel, the visible light images of size 512 pixel $\times 320$ pixel, and the natural images of size 768 pixel $\times 512$ pixel, respectively. Test results are shown in Table 1 . Among the five methods, the 2DHE method and the 2DFM method have the shortest processing time and the fastest processing speed, but the enhancement effects are not obvious; the BM3D method has the longest processing time; the processing time of the method proposed in [18] is shorter than the proposed method with the different sizes of images, but the enhanced images by the method proposed in [18] appeared fuzzy phenomenon. In order to improve this problem, we introduced a compensation measure factor to ameliorate the proposed method. The results of simulation and tests indicate that using the proposed method the noise the enhanced images can be removed more effectively while less calculation amount is increased. 
Table 1 Comparison of processing time with five methods

\begin{tabular}{cccc}
\hline & Infrared images & Visible light images & Natural images \\
Image size & 320 pixel $\times 256$ pixel & 512 pixel $\times 320$ pixel & 768 pixel $\times 512$ pixel \\
\hline 2 DHE $/ \mathrm{s}$ & 0.8 & 0.71 & 1.1 \\
$2 \mathrm{DFM} / \mathrm{s}$ & 0.68 & 0.5 & 0.51 \\
BM3D $/ \mathrm{s}$ & 110.03 & 226.41 & 535.35 \\
Method proposed in $[18]$ & 5.13 & 10.47 & 26.01 \\
$/ \mathrm{s}$ & 19.06 & 38.39 & 93.12 \\
\hline
\end{tabular}

\subsection{Impersonal Comparison}

In the previous section, we provided an intuitive comparison of image enhancement algorithms applied to infrared and visible images. We further illustrate the superiority of our proposed method via six evaluation values calculated on each test image to assess the enhancement effect and image quality: these six criteria are root-mean-square contrast (RMSC) ${ }^{26}$ local contrast, ${ }^{27}$ information entropy, fuzzy index, mean square error (MSE), and peak signal-to-noise ratio (PSNR), respectively.

In this letter, we chose 20 pictures of infrared images and visible images, respectively, as test images. 
Table 2 Statistical evaluation (MEAN) of 20 infrared images.

\begin{tabular}{cccccc}
\hline & 2DHE & 2DMF & BM3D & $\begin{array}{c}\text { method } \\
\text { proposed in } \\
{[18]}\end{array}$ & proposed method \\
\hline RMSC & 60.9983 & 43.3724 & 44.4207 & 42.1414 & 45.7532 \\
Contrast & 0.7873 & 0.1651 & 0.2578 & 0.1492 & 0.2613 \\
Entropy & 8.1585 & 8.2194 & 6.9962 & 8.0298 & 7.8274 \\
Fuzzy Index & 0.0807 & 0.0814 & 0.0828 & 0.0795 & 0.0565 \\
MSE & 91.5839 & 90.9607 & 74.3170 & 96.2064 & 144.7559 \\
PSNR & 28.5126 & 28.5423 & 29.4199 & 28.2988 & 26.5244 \\
\hline
\end{tabular}

Table 3 Statistical evaluation (MEAN) of 20 visible images.

\begin{tabular}{|c|c|c|c|c|c|}
\hline & 2DHE & $2 \mathrm{DMF}$ & BM3D & $\begin{array}{c}\text { method } \\
\text { proposed in } \\
{[18]}\end{array}$ & proposed method \\
\hline RMSC & 70.3846 & 52.2541 & 45.6903 & 45.9708 & 63.4006 \\
\hline Contrast & 0.7604 & 0.1385 & 0.3273 & 0.1025 & 0.3623 \\
\hline Entropy & 7.2222 & 7.1800 & 7.3955 & 7.1824 & 7.3097 \\
\hline Fuzzy Index & 0.1388 & 0.2225 & 0.1387 & 0.2348 & 0.1651 \\
\hline MSE & 232.1120 & 101.5583 & 80.7617 & 108.260 & 142.2839 \\
\hline PSNR & 24.4738 & 28.0637 & 29.0588 & 27.7861 & 26.5992 \\
\hline
\end{tabular}

For this letter, we selected 20 infrared and 20 visible images as test images; Tables 2 and 3

show the statistical results of these two sets of test images, respectively. Each number listed in the two tables is the mean of 20 images. We observe that RMSC and local contrast for our 
proposed method are slightly below that of 2DHE, though higher than that of 2DMF, BM3D, and the method proposed in [18]; however, the information entropy and fuzzy index of our proposed method are better than the other three methods. Estimating the ability to reduce noise via the two values of MSE and PSNR, our proposed method is lower than that of 2DHE for visible images, but higher than that of the other three methods. Overall, the comprehensive performance of our proposed method is better than that of the other three methods.

Note that the parameters of the method proposed in [18] are set according to [18]. If we can optimize these parameters, we may be able to obtain better results.

\section{Conclusion}

In this leter, we proposed a novel enhancement method based on lateral inhibition networks. We established an improved lateral inhibition network to realize enhancement effects for increasing contrast and making edges more prominent. Furthermore, we proposed a compensation factor to eliminate image fuzziness. Our experimental results showed that image contrast was effectively enhanced by our proposed method, and compared with other enhancement methods, the visual quality of the noise-reduced images generated by our proposed method was better. Moreover, the original information can be exactly recovered with indispensable textural details. Hence, our proposed method successfully enhanced image contrast. For future work, we plan to improve our 
proposed method's robustness and apply it to images obtained from optoelectronic theodolite to improve image visibility and clarity.

\section{Acknowledgment}

This work was supported by National Science Foundation of China (NSFC) (Grand No.61405191) and the Jilin Province Science Foundation for Youths of China (Grant No.20150520102JH).

\section{References}

1. X.J. Liu, Y. Chen, W.S. Wang, and Z. Liu, "De-noising algorithm of wavelet threshold for small target detection," Chin. Opt. 5, 248-256 (2012).

2. A.V. Bronnikov and G. Duifhuis, "Wavelet-based image enhancement in x-ray imaging and tomography," Applied Opt. 37, 4437-4448 (1998).

3. W.D. Zhao, Z.J. Xu, J. Zhao, H. Wang, and F. Wang, "Enhancement of infrared image details based on gradient histogram transform," Opt. and Precision Eng. 22, 1962-1968 (2014).

4. Z.P. Wu, M. Xuan, H.G. Jia, M.C. Zhu, L.L. Guo, and H. Liu , "Infrared image dynamic range compression contrast enhancement based on optimal mapping curve," Chin. J. of Lasers 40, 217-224 (2013). 
5. H.G. Xia, J.C. Zhang, X.L. Ji, F.Yan, and X. Cao, "A resolution enhancement method based on interpolation and extrapolation in digital holography,” Chin. J. of Lasers 41, 222-227 (2014).

6. Q. Zhou, "Image denoising based on regional division and dictionary learning," Xi'an: Xidian University (2013).

7. Z.G. Zhou, N. Sang, and X.R. Hu, "Global brightness and local contrast adaptive enhancement for low illumination color image," Optik-International Journal for Light and Electron Optics 125, 17951799 (2014).

8. X.Z. Han, and J. Zhao, "Enhancement of image texture and contrast combined with partial differential equation," Opt. and Precision Eng. 20, 1382-1388 (2012).

9. F.C. Sun, R.C. Yang, and S.P. Dai, "Extraction of image information from heavy background interferences-image processing by simulating the principle of visual lateral inhibition," Acta Electronica Sinica 4, 68-72 (1980).

10. C. Luo, M.R. Zhao, D. Wang, and K.X. Cao, "Bionic compound eye panoramic detection and tracking strategy," J. of Image and Graphics 18, 1637-1643 (2013).

11. H.Y. Wang, and Y.J. Li, "Application of lateral inhibition models in color image edge enhancement," Computer Engineering and Application 26, 55-57 (2003).

12. K.L. Gao, and F.Q. Zhou, "Cheng Wei, Adaptive inhibition method with double filtration to detect edge in IR sequence images," Microelectronics 24, 156-158,162 (2007). 
13. F. Wilkinson, H.R. Wilson, and D. Ellemberg, "Lateral interactions in peripherally viewed texture arrays,” J. Opt. Soc. Am. A 2057-2068 (1997).

14. M.H. Liang, "Lateral inhibition : inherent recurrent processes in coherent optical propagation," Applied Opt. 33, 158-161 (1994).

15. X.F. Xie, X.B. Mao, and T.J. Chen, "Study on image enhancing algorithm based on improved lateral inhibition," Computer Eng. and Applications 46, 148-150 (2010).

16. H.X. Liu , X.D. Yao, and Q.Chang, "Application of lateral inhibition network in image enhancement," J. of East China University of Science and Technology(Natural Science Edition), 33, 120-124 (2007).

17. Q. Jing, S.W. Xuan, and R.F. Wu, "Local fractional differential image enhancement based on lateral inhibition principle," Computer Eng. and Design 34, 2826-2833 (2013).

18. S.S. Dai, Q. Liu, P.F. Li, J.S. Liu, and H.Y. Xiang, "Study on infrared image detail enhancement algorithm based on adaptive lateral inhibition network," Infrared Phys. \& Technology 68, 10-14 (2015).

19. M.L. Shi, Z.M. Peng, Q.H. Zhang, Q.Z. Li, and Z.Q. Lin, "Dim infrared target detection based on adaptive lateral inhibition network," High Power Laser and Particle Beams 23, 906-910 (2011).

20. J.M. Geusebroek, A.W.M. Smeulders, and J.V.D. Weijer, "Fast anisotropic Gauss filtering," IEEE Trans. Image Process 12, 938-943 (2003). 
21. J. Wu, Y.Y. Wang, Y. Chen, J.H. Yu, and Y. Pang, "Speckle reduction of ultrasound images with anisotropic diffusion based on homogeneous region automatic selection," Opt. and Precision Eng. 22, 1312-1321 (2014).

22. X.F. Xie, "Research of image enhancement algorithm based on human vision characteristics," M.S. thesis, Dept. Electron. Eng., Zhengzhou Univ., Zhengzhou, China, 2009.

23. LIVE Image Quality Assessment Database Release 2[Online]. http://ive.ece.utexas.edu/research.

24. C. Turgay, "Two-dimensional histogram equalization and contrast enhancement," Pattern Recognition 45, 3810-3824 (2012).

25. M.C. Wang, L.X. Xing, Y.H. Yang, Z.H. Liu, "Directinal weighted two-dimensional multi-step median filtering in image processing," J. of Jilin University(Information Science Edition) 24, 1821(2006).

26. C. Zuo, Q. Chen, N. Liu, J. Ren, and X.B. Sui, "Display and detail enhancement for high dynamic range infrared images," Opt. Eng. 50, 127401 (2011).

27. B.C. Zhan, Y.Q. Wu, and S.X. Ji, "Infrared images enhancement method based on stationary wavelet transformation and retinex," Acta Optica Sinica 30, 2788-2793 (2010). 